\title{
Criação de um chatbot para atendimento de suporte sistêmico de uma rede de supermercados a partir de uma base de conhecimento registrada no Mantis ${ }^{\circledR}$
}

\section{Creation of a chatbot for the systemic support of a supermarkets chain from a knowledge base registered in the Mantis ${ }^{\circledR}$}

\author{
Viviâne de Almeida Tôrres ${ }^{1}$, Luiz Cláudio Gomes Maia ${ }^{2}$ e Cristiana Fernandes de Muylder ${ }^{3}$ \\ ${ }^{1}$ Universidade FUMEC - Belo Horizonte, Minas Gerais, Brasil. ORCID: http://orcid.org/0000-0001-8250-594X \\ 2 Universidade FUMEC - Belo Horizonte, Minas Gerais, Brasil. ORCID: http://orcid.org/0000-0002-2568-6067 \\ ${ }^{3}$ Universidade FUMEC - Belo Horizonte, Minas Gerais, Brasil. ORCID: http://orcid.org/0000-0002-0813-0999
}

Autor para correspondência/Mail to: Viviâne de Almeida Tôrres, viviane.almeida.torres@gmail.com

Copyright (c) 2019 Tôrres, Maia, \& Muylder. Todo o conteúdo da Revista (incluindo-se instruções, política editorial e modelos) está sob uma licença Creative Commons Atribuição-NãoComercial-Compartilhalgual 3.0 Não Adaptada. Ao serem publicados por esta Revista, os artigos são de livre uso em ambientes educacionais, de pesquisa e não comerciais, com atribuição de autoria obrigatória. Mais informações em http://revistas.ufpr.br/atoz/about/submissions\#copyrightNotice.

\begin{abstract}
Resumo
Introdução: a tecnologia está presente nos diversos ramos de atividades e somente ela não é capaz de ser um diferencial nas empresas. Faz-se necessário ter o domínio do negócio e ter o conhecimento sobre a área que está sendo avaliada. O conhecimento pode ser apresentado em diversos formatos, estando este intangível ou devidamente registrado. Quando há registro desse conhecimento, a tarefa de buscar essas informações pode ser realizada com facilidade e permite agilidade e eficiência para a tomada de decisão. No entanto, recuperar informações com precisão não é uma tarefa simples.

Metodologia: o presente trabalho trata sobre a geração de uma coletânea de documentos (corpus em formato adequado para (chatbots) criada a partir de uma base histórica de atendimentos do sistema Mantis $\mathbb{R}$ (software livre que permite o registro de bug's e melhorias) de uma rede de supermercados. A base foi submetida a um serviço de perguntas e respostas que possui técnicas de recuperação de informações, bot e conversação natural, a fim de realizar análise dos retornos recuperados.

Resultados: o resultado do trabalho é a conversão de vários registros de soluções já revisadas anteriormente, em um direcionamento único e objetivo, do que a leitura de diversas possibilidades de soluções dispersas em vários atendimentos. Os atendimentos são considerados como base de conhecimento para a recuperação de informações e a resposta para o usuário.

Conclusão: com isso, a pesquisa concluiu que o chatbot localiza respostas e consolida possíveis soluções utilizadas em atendimentos anteriores e que podem ser a solução do problema relatado atualmente. Os chatbots podem ser uma alternativa frente a necessidade de atender usuários na prestação de serviço de suporte.
\end{abstract}

Palavras-chave: Sistemas Perguntas e Respostas; Chatbot; Mantis $($; Recuperação da Informação; Rede de Supermercados.

\begin{abstract}
Introduction: technology is present in the various branches of activity and cannot be the only differential in companies. It is necessary to have mastery of the business and have knowledge about the evaluated area. Knowledge can be in different formats, whether intangible or properly registered. When this knowledge is recorded, searching for this information can be performed easily and allows agility and efficiency for decision-making. However, retrieving information accurately is not a simple task.

Method: The present work deals with the generation of a collection of documents (corpus in a format suitable for chatbots) created from a historical base of calls from the Mantis $(\mathbb{B}$ ) system (free software that allows bug registration and improvements) from a supermarket chain. The database was submitted to a question and answer service that has information retrieval, bot and natural conversation techniques, in order to perform analysis of the recovered returns.

Results: : The result of the work is the conversion of several records of solutions previously reviewed, in a single and objective direction, than the reading of several possibilities of solutions dispersed in several services. The services are considered as a knowledge base for information retrieval and the answer for the user.

Conclusions: thus, the research concluded that the chatbot finds answers and consolidates possible solutions used in previous visits and that might be the solution to the problem currently reported. Chatbots can be an alternative in view of the need to assist users in providing support services.
\end{abstract}

Keywords: Questions and Answers Systems; Chatbot; Mantis $\mathbb{R}$; Information Retrieval; Supermarket chain.

\section{INTRODUÇÃO}

Segundo os autores Lopes, Muylder, e Judice (2011), a informação é um ativo organizacional em um cenário em que a competitividade, as inovações tecnológicas e a diminuição da distância tornaram o processo de tomada de decisão e o tempo diferenciais diante da concorrência. As boas práticas da Governança da Tecnologia de Informação classificam a informação como um ativo da Tecnologia de Informação que deve ser gerenciado de forma segura nas empresas para que possam agregar valor ao negócio (Puhl \& Preuss, 2013).

Segundo Parreiras e Bax (2003), o volume de informações e de dados criados nas empresas de médio e grande porte para utilização interna e externa é espantoso. Trata-se de um volume muito alto de documentos dentre relatórios, websites e publicações. Com isso, é necessário avaliar sobre o armazenamento dessas informações, 
a sua recuperação, uma gestão de conteúdo eficiente para centralizar os dados e liberar acesso aos indivíduos autorizados.

Perguntas objetivas e respostas rápidas também são índices de aumento entre os sites, visando direcionar os interessados mediante volume elevado de conteúdo distribuído. Segundo Roy et al. (2018), o site de perguntas e respostas da CQA Community Question Answering (CQA) tornou-se um local de grande visibilidade entre os usuários da internet. Ainda de acordo com os autores, o Stack Exchange é um dos sites mais populares do CQA, onde o conteúdo é publicado todos os dias na forma de perguntas, respostas e comentários. As respostas são listadas por suas ocorrências recentes, tempo de postagem ou votação.

Assim como a web, o volume de informações nas empresas cresce exponencialmente, e administrar o volume de informações que trafegam nas redes internas e externas tornou-se uma preocupação. O excesso de dados ou sua redundância ou a dificuldade de localização prejudicam a produtividade das organizações (Núcleo Health Care, 2017). Realizar pesquisas assertivas, recuperar regras de negócios e localizar informações consideradas pela empresa com alto teor de importância de maneira rápida e ágil para tomadas de decisões também se tornou uma situação cada vez mais recorrente nas Organizações.

Diante desse cenário, busca-se compreender o seguinte problema de pesquisa: como responder de maneira assertiva e rápida as dúvidas e questionamentos de usuários a partir de uma base histórica em uma rede de supermercados frente a necessidade diária de atendimento de suporte a sistemas?

Este artigo tem como objetivo propor e avaliar os retornos (respostas) de um chatbot (programa que faz parte da linha de sistemas de perguntas e respostas, que obtiveram grande avanço dentro da inteligência artificial por representarem o conhecimento e o processamento natural) às perguntas inseridas em formato de linguagem natural, utilizando uma coletânea de documentos (corpus) gerada a partir de um banco de dados de 7 GB de histórico de atendimentos já existente com um domínio específico. Trata-se de uma base de atendimentos de suporte sistêmico de uma rede de supermercados da região de Belo Horizonte, que foram registrados no software Mantisß).

\section{CONTEXTUALIZAÇÃO DO AMBIENTE}

A rede de supermercados que este artigo utilizou como estudo é a Organização Verdemar Ltda., que atende a diversas regiões da Região Metropolitana de Belo Horizonte e município de Nova Lima com foco nos clientes de classe A e B e com atuação desde 1993. Trata-se de uma rede com mais de vinte dois mil produtos, entre nacionais e importados, além dos produtos de fabricação própria, doravante denominada apenas por Verdemar. O Verdemar é portador de vários prêmios e, segundo o site Supermercado Moderno (2017), no ano de 2016, ocupou a quadragésima quarta posição em nível nacional, oitava em nível estadual e trigésima primeira em nível de tipo de loja igual a supermercados.

O Verdemar possui o setor de Tecnologia de Informação (TI) com cerca de 31 colaboradores e os sistemas que auxiliam na gestão empresarial é de desenvolvimento próprio, ou seja, existe uma equipe de desenvolvimento e testes de software que analisa possíveis falhas e customizações inerentes à implementação dos sistemas de informações, que atendem aos diversos setores da empresa (Verdemar, 2017). Apesar de a empresa investir em Tecnologia da Informação e adotar o sistema descrito como ferramenta para gerenciar os processos internos do Setor de TI, entende-se que o volume de dados é crescente. A própria Organização, ao longo de 24 anos, registrou sua expansão de duas lojas para 12 lojas atualmente, com planejamento futuro para novas unidades, o que requer um esforço da atualização da tecnologia. Os softwares, de maneira geral, requerem contínua avaliação e desenvolvimento frente as mudanças de mercado.

A área da Tecnologia da Informação está dividida em quatro células para atender de maneira eficaz as demandas da empresa, são elas: Suporte Técnico, Suporte a Sistemas, Infraestrutura e Desenvolvimento Interno. As áreas possuem interação entre elas, no entanto sua subdivisão permite a especialização dos atendimentos do dia a dia e nos projetos de melhorias.

A célula de Suporte Técnico é responsável por instalar, manter, controlar e acompanhar equipamentos, máquinas e acessórios que dão subsídio à execução de tarefas administrativas e operacionais das lojas. Os serviços variam desde a formatação de uma máquina até a configuração de um coletor de dados que executa operações como inventários e validação de preços. A célula possui como principal objetivo manter a disponibilidade do ponto de venda para que os clientes efetuem suas compras. A equipe é dividida entre as lojas para garantir que os equipamentos estejam em bom funcionamento.

A Tecnologia da Informação sistêmica possui grande importância no cenário competitivo das empresas, principalmente quando tornam os processos mais ágeis, transparentes e eficientes. Cada vez mais, os resultados das empresas estão sendo analisados minuciosamente, visando encontrar falhas para correção, redução de falhas já identificadas e maximização dos lucros. Para isso, a gerência por meio de sistemas é cada vez mais utilizada. 
Os sistemas permitem análise dos gestores e tomadas de decisões, baseadas no cenário que o sistema apresenta e na interpretação da gestão. Nenhum sistema substitui o feeling e a experiência de um ser humano, no entanto, a visão que o sistema possibilita permite ao responsável direto inferências mais assertivas.

O Verdemar possui desenvolvimento interno e próprio para atender as demandas da empresa de maneira ágil e em conformidade com as inovações que são consideradas como diferenciais para o negócio. Atendimento à legislação e a processos contábeis e burocráticos são implementados como rotinas que visam informatizar, melhorar o controle, criar histórico para tomada de decisões e contribuir para o constante aperfeiçoamento dos processos da empresa.

A célula de infraestrutura é responsável por todo o arcabouço tecnológico da empresa, proporcionando um conjunto de elementos que dão suporte às áreas de suporte técnico, suporte a sistemas e para o desenvolvimento, assim como a empresa como um todo. Realiza monitoramento, gerência e configuração da rede de dados, controle de equipamentos de telecomunicação e tráfego de transações. É responsável por desenvolver soluções lógicas a partir de recursos de hardware/software para rede.

\section{Mantis $\mathbb{R}$ Sistema de Registro de Bug's e Melhorias}

Os sistemas que auxiliam na gerência dos processos e atividades da empresa são desenvolvidos internamente. Por esse motivo, fez-se necessário gerenciar as possíveis falhas e customizações de melhorias para manutenção desses sistemas e acompanhar as novas demandas. O controle atual é gerenciado pelo software Mantis@). O Mantis (R)atende ao departamento de TI do Verdemar frente as necessidades de organização e gestão das solicitações feitas pelos clientes internos. Trata-se de um sistema livre que permite gerenciar projetos de software tendo código aberto para modificações. Ele possui notificações de e-mail, controle de acesso, abertura de "casos" (relatos para verificação com a possibilidade de criar fluxos de processo), pesquisas de casos ativos e encerrados, gerenciamento de itens na agenda de análise, controle de registros e acessos, entre outras funcionalidades.

O Mantis@foi implantado na versão 1.2.9 desde meados de 2010, utilizando a plataforma de linguagem PHP com implementação no banco de dados MySQL Versão 5.0. A partir dessa implantação não houve atualizações posteriores devido sua utilização intensa. Os dados registrados no software Mantisßforam utilizados para análise da pesquisa, considerando que sua utilização proporciona ao Setor de TI a possibilidade de pesquisar informações sobre a implantação de projetos anteriores, assim como falhas identificadas de software, análise de documentações, verificação de Definição de Requisitos de Usuários de (DRU) e informações diversas sobre os sistemas da empresa. Quaisquer informações relativas a sistemas estão documentadas em um caso (registro) desse sistema e as informações são centralizadas permitindo consultas e visualização do histórico dos dados, auxiliando na resolução de problemas atuais já vividos anteriormente e na análise de problemas parecidos ou na análise de projetos.

No entanto, o volume de informações, dados e documentos inseridos no Mantisßaumenta diariamente, e essas informações registradas servem de auxílio para realização dos atendimentos diários, feitos pelos clientes internos, relatando sobre dúvidas e possíveis falhas. Ao tentar pesquisar assuntos já registrados, a quantidade de itens retornados é grande, dificultando um atendimento rápido.

Cada projeto possui uma classificação separada no Mantis $\AA$, na qual os registros referentes a ele são feitos. Existem casos com situação aberta, resolvida ou até mesmo fechada. Todos os registros servem de auxílio para realizar os atendimentos, principalmente os registros com situação fechada, que possuem a informação do que foi feito para finalizar o atendimento que, no Mantisß), é chamado de caso (similar a chamado, solicitação). Os principais projetos utilizados atualmente somam um total de mais de 19.000 registros (casos) em um período de seis anos. Para cada caso aberto, os dados do atendimento são inseridos de acordo com as informações repassadas pelos clientes internos e posteriormente são utilizados como histórico para solucionar outros problemas similares.

\section{Cenário atual}

O sistema de registro de bug's e melhorias no Mantis@auxilia no controle dos atendimentos e casos, no entanto, sua pesquisa é limitada em filtros pré-determinados ou em consultas de texto livre, o que permite que vários casos sejam retornados e que seja necessário analisar manualmente qual registro se refere ao atendimento que está sendo feito no momento da solicitação do cliente. Com isso, o atendimento se torna moroso e lento. Uma pesquisa é feita utilizando palavras-chave ou os filtros padrões do sistema, como por exemplo: uma nota fiscal apresenta problemas relativos ao ICMS (Imposto sobre Circulação de Mercadorias e Serviços), conforme ilustra Figura 1.

O exemplo simula um cenário real em que o cliente interno entra em contato, informando que a nota fiscal que está sendo recebida nos depósitos das lojas apresenta problemas relativos ao ICMS, um tributo pago ao Governo e que precisa ser apurado pelo sistema. Para orientar o cliente sobre o procedimento correto a ser feito, ou até mesmo para verificar se o atendimento se trata de uma possível falha no sistema ocorrida outras vezes, o analista realiza pesquisas na base histórica Mantis $囚$. 
São feitas duas consultas ao sistema Mantis®pela palavra-chave "nota fiscal ICMS" (Figura 1), sendo que o primeiro filtro está sem o campo categoria selecionado e o segundo especifica que são somente registros classificados como falha. Na primeira consulta, 392 registros são retornados para verificação do analista e na segunda 121 registros. Em ambos os casos, o resultado é excessivo diante da pergunta inserida. Mesmo inserindo mais informações na consulta, o analista apresenta dificuldades em localizar uma resposta que o auxilie no atendimento que, em sua maioria, são feitos pelo telefone.

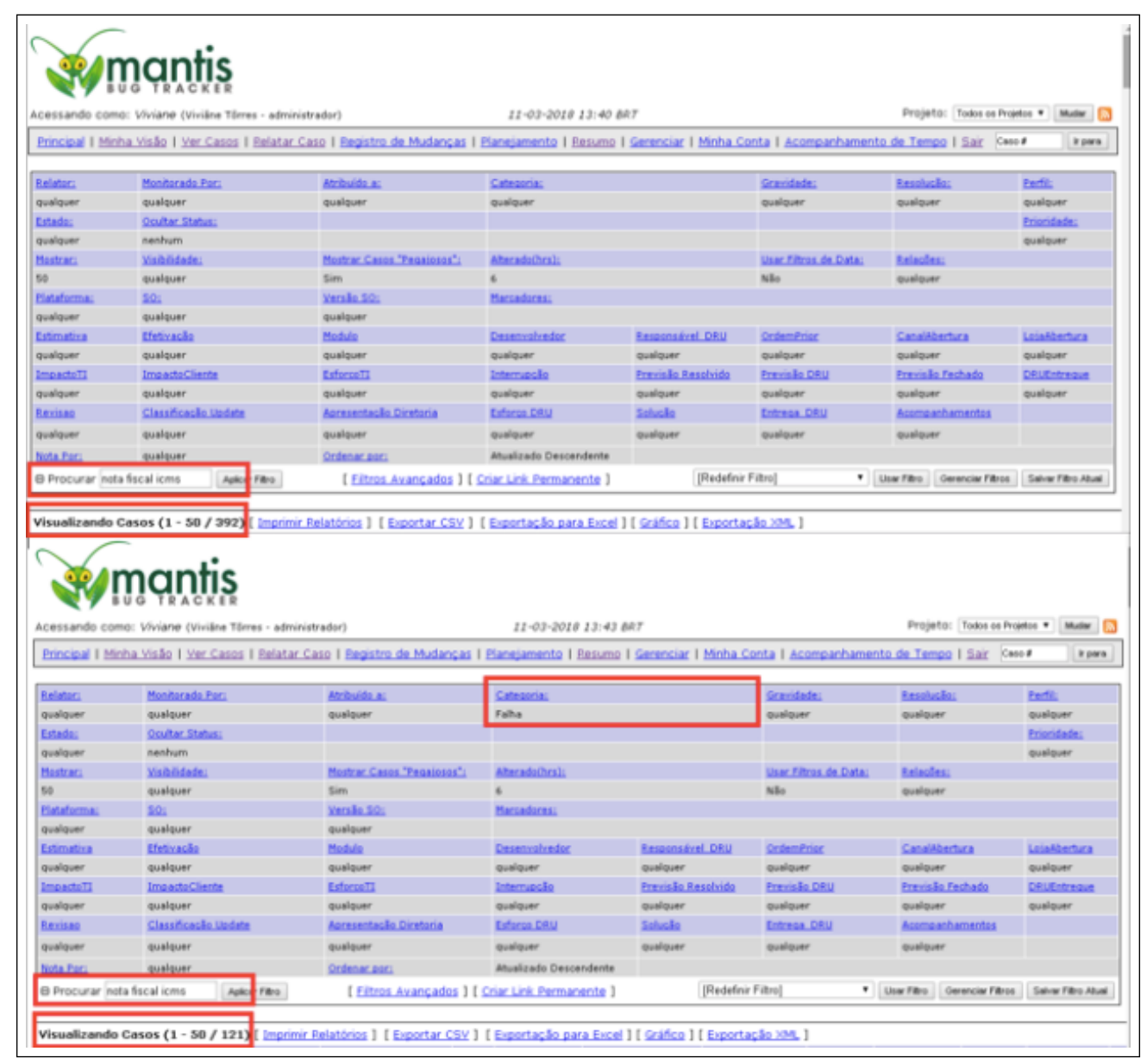

Figura 1. Tela de pesquisa do Mantis $\mathbb{R}$

Fonte: Verdemar (2017).

O tempo de espera da resolução do problema de uma nota fiscal acarretará em atrasos no descarregamento da mercadoria e com isso afetará a disponibilidade do produto na área de venda, ou seja, uma possível ruptura que foi destacada como um problema comum nos supermercados. Para evitar esse problema, outro pode ser gerado quando a equipe de suporte a sistemas não realiza a análise e aciona a equipe de desenvolvimento para avaliar diretamente no código fonte o problema informado pelo cliente que, ao final da verificação, pode concluir por uma falha operacional de digitação. Trata-se de um problema em cadeia.

\section{Cenário desejado}

O registro dos atendimentos no sistema Mantis@é fundamental para a criação da base histórica de suportes já realizados para controle das demandas atendidas e para permitir a consulta posterior de soluções utilizadas na resolução dos problemas identificados. No entanto, espera-se que essa consulta seja breve, de fácil acesso e de boa usabilidade. Perguntas completas podem permitir maior agilidade nos atendimentos telefônicos, remotos e presenciais, visto que os filtros padrões disponibilizados pelo sistema restringem as pesquisas.

A possibilidade de digitar uma pergunta e o sistema já apresentar todas as possíveis soluções para o problema informado é diminuir o tempo de análise e o tempo por parte da equipe de suporte, diminuir o tempo de espera da solução por parte do cliente, otimizar o atendimento e proporcionar maior rapidez e interatividade nos atendimentos. Espera-se maior flexibilidade do sistema, porque até mesmo os analistas de suporte possuem dúvidas nos atendimentos ou estão mais capacitados em outros tipos de atendimentos e assuntos específicos. 


\section{METODOLOGIA}

A pesquisa apresentada neste artigo foi dividida em sete etapas, de acordo com os objetivos do trabalho, com o intuito de atingir o objetivo geral de testar e/ou aplicar uma das técnicas de sistemas de perguntas e respostas em uma base histórica, a fim de realizar análises dos resultados recuperados avaliando sua assertividade. O sistema Mantis@possui MySQL (um dos componentes centrais da maioria das aplicações públicas da Internet) como gerenciador do seu banco de dados. A base utilizada possui os atendimentos registrados até Abril/2018, cerca de 19.200 atendimentos. Esse histórico dos atendimentos resulta em aproximadamente 7 GB de tamanho. O sistema Mantis@ utiliza a linguagem de script open source PHP (um acrônimo recursivo para PHP: Hypertext Preprocessor) para aplicação do Mantisß. Os dados inseridos no Mantis@até Abril/2018 foram copiados para a criação de dump (registro da estrutura da tabela ou dados de um banco de dados) para armazenar uma cópia de segurança e realizar os experimentos por meio dos dados registrados. As sete etapas são:

A primeira etapa é a Exportação dos Dados do Mantis@e Extração de Tabelas: Os dados que estão registrados no Mantisßforam exportados em tabelas que serão úteis para a realização do trabalho. Realizou-se o procedimento de verificação de quais dados e tabelas são relevantes para o trabalho, utilizando o banco de dados MySQL. O procedimento foi feito por meio de comandos de "backup" fornecidos pelo próprio gerenciador de banco de dados MySQL. Logo após foi feito "restore" deste backup em uma outra máquina, com um novo banco de dados MySQL (o mesmo utilizado anteriormente), a análise de quais tabelas seriam úteis e relevantes para o trabalho foi feita manualmente. Somente as tabelas que possuem as informações dos atendimentos foram selecionadas; sendo elas:

- mantis_bug_table;

- mantis_project_table;

- mantis_category_table;

- mantis_bug_text_table;

- mantis_bugnote_table;

- mantis_bugnote_text_table.

Os dados foram extraídos destas tabelas por meio de sentenças SQL (Structured Query Language), o que possibilitou a geração bruta da informação utilizada posteriormente. Para elaboração das perguntas e respostas do sistema a ser desenvolvido, os principais campos utilizados foram: ID do caso (número de registro no sistema, autoincremental), data de inclusão, categoria (trata-se da classificação do caso: melhoria, falha, select, banco de dados, entre outros), projeto (trata-se da descrição do projeto, uma subdivisão, pode haver vários projetos registrados), resumo (trata-se de exposição sucinta da solicitação) e anotações (andamento da solicitação até sua resolução final). As tabelas foram classificadas como relevantes porque possuem a descrição do problema e o que foi feito para solucioná-lo. De modo geral, essas tabelas possuem os metadados: ID do Caso, Resumo, Categoria e Anotações. Nesses campos estão descritos os problemas informados pelos usuários e a solução repassada pelo analista.

A segunda etapa é a Montagem do Corpus. Utilizou-se o banco de dados do sistema Mantisß, que é o software utilizado na Organização Verdemar Ltda. Foi feita a conversão do banco de dados do sistema Mantisß)(que possui os registros de atendimentos) para o formato. TXT. Adotou-se o processo de conversão dos dados, mapeamento das tabelas e select's (declaração SQL que retorna um conjunto de resultados de registros de uma ou mais tabelas) das possíveis respostas por meio do conceito de ETL, do inglês Extract Transform Load (Extração Transformação Carregamento) do software PDI (Pentaho Data Integration, 2018), fornecido pela Hitachi.

Logo após foi feita a Elaboração das Perguntas x Repostas. A elaboração dos questionamentos para serem inseridos no sistema, tendo as respostas como referência para avaliação dos testes, foram elaboradas por meio do o serviço da Microsoft Azure (2018) por meio da API Microsoft QnA Maker (serviço de perguntas e respostas com uma interface gráfica) para elaborar as perguntas a partir do conteúdo da base já existentes (Mantis@).

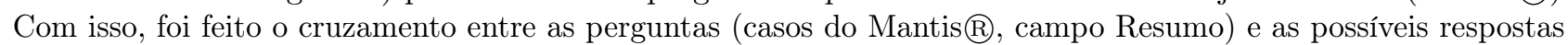
(campo Anotações) em uma tabela temporária.

Na quarta etapa, foi feita a Retirada de Caracteres Especiais ou Caracteres Inválidos. Os caracteres especiais ou inválidos que atrapalham a verificação do contexto foram removidos, utilizando uma função do software PDI (Pentaho Data Integration, 2018) para esse procedimento. Com isso, foi possível dar sequência e realizar o processo de Montagem do Arquivo Final, em extensão.TXT, que será importado ao serviço do QnA Maker na plataforma Microsoft Azure, gerando uma base de conhecimento com o formato de par de chaves (key pair; Pergunta: Resposta), concluindo a quinta etapa. Após a importação do arquivo, o QnA Maker gera várias perguntas e suas possíveis respostas. Nesse momento é conferida manualmente a relevância do key pair (confidence score) para melhorar a assertividade da ferramenta e a criação do ranking das respostas. O QnA Maker permite realizar inspeções nas perguntas e respostas para que os ajustes sejam realizados, acrescentando perguntas alternativas, porém com a mesma resposta (solução do atendimento). 
De posse do arquivo gerado, é necessário treinar o sistema. Sexta etapa, Adequação das Respostas (Treinamento). Foi realizada a análise das relações entre as perguntas e a relevância das respostas, para que o ranking das possíveis respostas esteja mais coerente ao esperado. Foi feita análise manual para o sistema "aprenda" a melhor resposta. Etapa final, a Análise das Respostas. As respostas retornadas para cada pergunta. Os resultados retornados foram analisados manualmente.

\section{TESTES E RESULTADOS OBTIDOS}

Os testes realizados comprovam a autenticidade da ferramenta QnA Maker dentro dos propósitos que se designa a realizar. Possui interação amigável com o usuário, apesar de não ter sido localizada a possibilidade para alterar o idioma. Seus passos para criação da base de conhecimento são sequenciais e intuitivos, as telas possuem layout's de fácil aprendizado. Suas funções são parametrizáveis, o que permite maior número de possibilidades para criação de avaliações e exames.

A ferramenta Microsoft Azure possui várias possibilidades de configuração e parâmetros, o que permite testes e ajustes para novas verificações, além de permitir parametrizações de perfil para permissões, recursos para assinatura (compra de licença), foto do usuário, idioma preferido entre outros.

Os testes foram realizados inserindo perguntas relativas a notas fiscais eletrônicas, impostos, atividades processuais do dia a dia de um supermercado (alterações de preço de venda, pedidos de compra, entregas de mercadorias, vendas, emissão de documentos, entre outros) e itens específicos da empresa objeto de estudo como atividades de ruptura, atividades de importação de produtos entre outros, e o sistema apresentou as respostas de acordo com o histórico de anotações importado e com o ranking criado pelo QnA Maker.ai.

Os retornos apresentados pelo sistema foram satisfatórios, as respostas automáticas foram apresentadas com tempo de resposta muito rápido, em torno de um a dois segundos, apresentando bastante coerência com a pergunta realizada, podendo auxiliar a equipe de suporte mediante os atendimentos do dia a dia de forma mais assertiva e rápida. No entanto, as respostas são apresentadas na íntegra de sua anotação, sendo necessário refinar da base para tornar as respostas mais amigáveis e naturais. Atualmente a resposta correta e mais próxima da solução definitiva é apresentada de maneira direta sem gentileza ou qualquer cortesia. Veja o exemplo:

Usuário: "Por que o sistema não apresentou trava de alerta para emissão de pedido de transferência?" Sistema: "Após a análise, foi identificado que: No cadastro da operação 162. Na aba itens a flag - Apenas produto de insumo está marcada, juntamente com a flag "Transferir IND sem receita na seção marcada", na sub aba 'Bloqueia". Isso impede que o produto seja transferido para a seção se ele não possuir receita nesta seção." Análise: Tratava-se de uma configuração na operação.

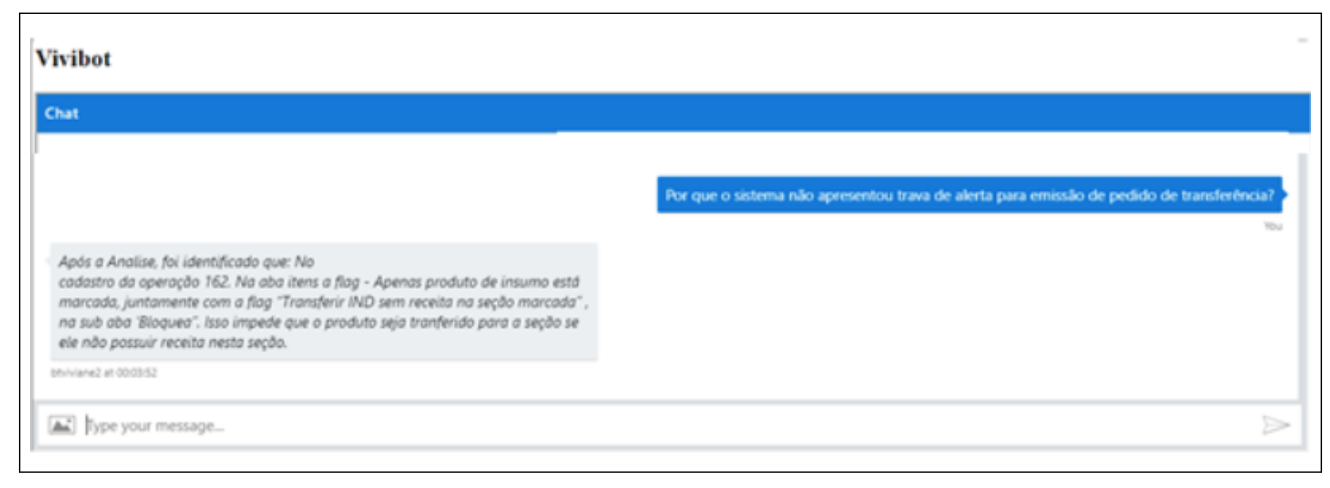

Figura 2. Pergunta 1 - Tela do Chatbot Fonte: Microsoft Azure (2018).

As respostas estão corretas e podem ser utilizadas, principalmente, para os atendimentos telefônicos que requerem maior agilidade.

Notou-se lentidão para importação dos dados e para a publicação do chatbot. Com isso, para validação de parâmetros/configurações e desempenho da internet (trata-se de uma ferramenta online) para fins de testes, foi importada uma base com tamanho correspondente a um quarto da base completa (aproximadamente 2 GB) para avaliação e foi detectado que a lentidão se manteve durante todas as etapas: importação dos dados, publicação, testes e inspeção. No entanto, a lentidão não foi vista como dificultador porque o tempo foi considerado como aceitável para edições e gravação dos dados.

O refinamento das perguntas e das anotações é imprescindível para que o sistema apresente desempenho cada vez melhor. O Mantis@é utilizado como um sistema de interação entre as equipes de suporte e desenvolvimento, logo, seu refinamento é algo moroso porque um caso (atendimento registrado) pode ter 1 para $\mathrm{N}$ anotações, ou 
seja, cada atendimento pode ter de uma a várias anotações, sem limite, sendo essas anotações irrelevantes para a solução definitiva do problema.

Em todos os atendimentos, foram retiradas as anotações referentes a: interação entre as equipes (suporte e desenvolvimento), solicitações de prioridade e urgência, solicitações de detalhamento e cenários de testes, informações de select's, alinhamentos internos e solicitações de autorização para encerrar o atendimento após solução final ser apresentada. Trata-se de informações relevantes ao andamento do atendimento e não sobre a solução do mesmo.

Também foram identificados registros que poderiam ser expurgados de forma íntegra por não apresentarem importância na base de conhecimento, registros com classificação de "Diário" para apontamento de horas e identificação de tarefas, registros de solicitação de alterações via banco de dados, registros para criação de relatórios avulsos para análise. Esses registros são referentes a formalidades de atendimentos e poderiam ter sido localizados de acordo com a "Categoria" (campo padrão do Mantis@que permite classificar o atendimento), e serem desconsiderados no momento inicial da montagem do corpus.

Observou-se que houve a criação recente do campo personalizado "Solução" no Mantisße que poucos atendimentos (casos registrados) possuem o campo preenchido. Este campo foi desconsiderado para a criação da base por falta de documentação, no entanto, sua utilização aumentaria consideravelmente o desempenho do chatbot. Caso o campo "Solução" seja revisto e alimentado, o arquivo pode ser submetido novamente e criado uma nova base de conhecimento.

O QnA Maker, por meio de processamento interno, retorna a resposta mais aproximada, no entanto, seria de suma importância para o ranking de análise e para apresentar a solução imediata para novos atendimentos, sem necessidade de interação com uma outra equipe ou mesmo de consultas no Mantisß, que possui restrição de filtros pré-determinados. O campo "Resumo" foi utilizado como pergunta e o como "Anotações" como possíveis respostas. Foi identificado que a informação inserida no campo Resumo não é inserida em formato de pergunta, e sim como uma síntese do problema. Com isso foi necessário realizar ajustes para melhor apresentação.

Contudo, os testes foram aprovados porque as respostas realizadas ao chatbot foram respondidas com a solução correta para o questionamento. Para essa ação, foi feita comparação com a solução indicada no atendimento do sistema Mantis@. São necessários refinamentos para que a aplicação interaja de maneira cortês com o usuário e de forma mais natural, simulando um agente virtual com características sociáveis.

Sob o referencial teórico, notou-se que a informação pode ser considerada como um bem imprescindível para as empresas. O seu registro no sistema Mantisße, posteriormente, sua apresentação no chatbot, demonstrou o valor efetivo no esclarecimento de dúvidas inseridas durante os testes, conforme afirmado pelos autores Lopes et al. (2011) que a informação é um ativo organizacional. Assim como o seu crescente volume nos últimos anos, tanto na web quanto nos ambientes organizacionais segundo F. F. Almansa (2017) mediante o número de atendimentos registrados. Também foi identificado durante os testes que realizar o cruzamento das perguntas e das respostas dentro da base de conhecimento para organizar as informações e disponibilizar no chatbot, (retirando os dados desnecessários e duplicados), não é uma tarefa simples, como relatado por Souza (2006) quando afirma que a recuperação das informações possui dificuldades intrínsecas ao conceito de informação (F. F. Almansa, 2017, p.20).

Além disso, é possível verificar que os resultados estão em conformidade com os objetivos e baseados no referencial teórico proposto. Espera-se que os estudos sobre sistemas de perguntas e respostas sejam cada vez mais ampliados porque assim como abordado por L. Almansa e Macedo (2016), no ambiente médico, Moreno, Manfio, Barbosa, e Brancher (2015) no ambiente de comunicação instantânea, Leonhardt, Castro, Dutra, e Tarouco (2003) e Bada e Menezes (2012) no ambiente educacional, entre muitos outros autores, a busca por informações importantes e relevantes é uma tarefa que exige tempo e experiência do profissional para localizar respostas rápidas, e que podem ser auxiliadas pela tecnologia de informação por meio desses sistemas.

\section{CONLUSÕES}

A base histórica utilizada nos serviços da Microsoft Azure e QnA Maker para tratamento dos dados possibilitou responder ao questionamento inicial deste estudo. O sistema de chatbot permitiu interagir com o usuário e responder suas dúvidas de forma automática, retornando todas as possíveis soluções localizadas na base com tempo de resposta em torno de dois a cinco segundos, chegando a alguns casos até 20 segundos em média, diminuindo o tempo de atendimento manual, assim como as pesquisas manuais e o número de atendimentos realizados por atendentes, que gastam em média cinco minutos, os quais podem utilizar tempo ainda maior para análise dos resultados encontrados na base histórica.

A criação do chatbot, mesmo em caráter de pesquisa, demonstrou contribuição na entrega de respostas dos atendimentos na rede de supermercados, diminuindo a verificação manual das possíveis soluções aos questionamentos realizados, diminuindo o tempo de espera de minutos para segundos com possibilidade de aperfeiçoamentos 
futuros por meio de novos refinamentos e inspeções. Percebeu-se que retornar respostas que se aproximam da expectativa do solicitante (atendentes que realizaram a avaliação manual da resposta indicada pelo chatbot como possível solução) depende diretamente dos refinamentos e inspeções executados. Além disso, o tamanho da base histórica e o volume de informações podem afetar desempenho do tempo de resposta e a assertividade dos retornos automáticos. Recuperar a informação é uma necessidade real e não mais uma tendência ou um modismo. Verificou-se, durante a pesquisa, que o sistema de perguntas e respostas permite índice elevado de assertividade, uma vez que passa pelo processo de inspeção manual das respostas e pode ser reutilizado várias vezes retirando a interação com o atendente.

Conclui-se que, para responder automaticamente as dúvidas de usuários, em qualquer área de conhecimento, os chatbot's podem contribuir para realizar atendimentos, localizando informações de forma eficiente ou atendendo diretamente o usuário. Os chatbot's, quando refinados e preparados, são uma alternativa promissora para os atendimentos. Os objetivos do presente trabalho foram concluídos, avaliando manualmente os retornos apresentados pelo chatbot (Figura 2) após inserção de cerca de mais de 200 perguntas e assertividade de cerca de $70 \%$ nos retornos. Para aumentar cada vez o percentual de assertividade nos retornos, é necessário maior número de inspeções no QnA Maker. Não há somente técnicas como serviços preestabelecidos com procedimentos nativos de aprendizado de máquina e inteligência artificial que favorecem a implementação de sistemas de perguntas e respostas.

Consideram-se o experimento e o estudo de caso satisfatório, visto que os dados e informações crescem exponencialmente, e não há paralisações para a elaboração de conteúdo, crescendo também a complexidade de sua manipulação e extração de informações relevantes para tomadas de decisão. Os sistemas de perguntas e respostas, assim como os chatbots, são uma possibilidade de solução. O estudo permitiu avanços nas análises e nos testes das ferramentas que possuem alta capacidade de aproveitamento, com possibilidades reais de agilidade e diminuição de atendimentos, item muito relevante em um setor de suporte. O sistema confirma sua utilidade, sendo possível reproduções em ambientes de áreas diversas, sendo necessário um especialista do assunto para realizar avaliações.

\section{Limitações da pesquisa e recomendações para trabalhos futuros}

As limitações de pesquisa são referentes à tarifação do serviço e ao tamanho da base que dificultaram maiores refinamentos. Faz-se necessário o aprofundamento nos demais recursos que a ferramenta disponibiliza, entretanto, podem ser feitos posteriormente e ficando assim como sugestão futura. Como estudo futuro sugere-se uma revisão para preenchimento do campo solução do sistema Mantis@ $\mathbb{p}$ para os registros antigos e para os novos atendimentos. Logo após essa ação, sugere-se uma nova submissão da base de conhecimento com avaliações das equipes de suporte e de desenvolvimento da Organização. Por se tratarem de áreas com funções e objetivos distintos, apesar da parceria e interação, a comparação de percepção entre as equipes e a mensuração do número de assertividade do chatbot permitirão a criação de estatísticas para medir o nível de acertos e a eficiência das respostas. Além disso, sugere-se aprofundar nos recursos dos serviços Microsoft Azure e QnA Maker para testes e aplicações para a publicação do chatbot além de realizar testes apenas implementando a técnica Seq-to-Seq, utilizada pela ferramenta. 


\section{REFERÊNCIAS}

Almansa, F. F. (2017). Uma arquitetura de questionanswering instanciada no domínio de doenças crônicas. (Dissertação de mestrado). Universidade de Sã Paulo (USP), Brasil.

Almansa, L., \& Macedo, A. (2016). Sistema de informação para perguntas e respostas em doenças crônicas. In Anais principais do workshop de informática médica. Porto Alegre, Brasil. doi: 10.5753/sbcas.2016.9905

Bada, E. M., \& Menezes, C. S. (2012). Uma proposta para extração de perguntas e respostas de textos. In Anais do congresso internacional de informática educativa. Recuperado de http://www.tise.cl/volumen8/TISE2012/06.pdf.

Leonhardt, M. D., Castro, D. D., Dutra, R. L. S., \& Tarouco, L. M. R. (2003). Elektra: Um chatterbot para uso em ambiente educacional michelle. Renote: Novas Tecnologias na Educação, 1(2), 01-11. doi: doi.org/10.5753/CBIE.SBIE.2003.81-88.

Lopes, B., Muylder, C. F., \& Judice, V. M. M. (2011). Inteligência competitiva e o caso de um arranjo produtivo local de eletrônico brasileiro. Perspectivas em Ciência da Informação, 12(2), 213-231. Recuperado de http://www.spell.org.br/documentos/ver/912/ inteligencia-competitiva-e-o-caso-de-um-arranjo-produtivo -local-de-eletronica-brasileiro/i/pt-br.

Microsoft Azure. (2018). Api do qna maker. microsoft azure. Recuperado de https://azure.microsoft.com/pt-br/services/ cognitive-services/qna-maker/.

Moreno, F., Manfio, E., Barbosa, C. R., \& Brancher, J. D. (2015). Tical: Chatbot sobre o atlas linguístico do brasil no whatsapp. In Anais do simpósio brasileiro de informática na educação (sbie 2015). doi: https://doi.org/10.5753/cbie.sbie.2015.279.

Núcleo Health Care. (2017). Saiba como lidar com o excesso de informação nas empresas. (Mai, 10 ed.). Recuperado de http://nucleohealthcare.com.br/blog/2017/05/10/saiba -como-lidar-com-o-excesso-de-informacao-nas-empresas/.

Parreiras, F. S., \& Bax, M. (2003). A gestão de conteúdos no apoio à engenharia de software. In Anais do kmbrasil. Recuperado de https://www.dropbox.com/sh/ 6c1v19ja581lc8e/AADPKf8_2F-oUzc5S1mvQ7_oa/2003/ $\mathrm{GCT} ? \mathrm{dl}=0$ \&preview $=\mathrm{GCT} 142 . \mathrm{pdf}$

Puhl, R. L., \& Preuss, E. (2013). Gerenciamento de conteúdo corporativo (ecm) na gestão de ti. Encontro Anual de Tecnologia da Informação e Semana Acadêmica de Tecnologia da Informação, 3(1), 61-68. Recuperado de http://www .eati.info/eati/2013/assets/anais/AnaisEATI2013.pdf.

Roy, P., Ahmad, Z., Singh, J., Alryalat, M., Rana, N., \& Dwivedi, Y. (2018). Finding and ranking high-quality answers in community question answering sites. Global Journal of Flexible Systems Management, 19(1), 53-68. Recuperado de https://link.springer.com/article/10.1007/ s40171-017-0172-6.

Souza, R. R. (2006). Sistemas de recuperação de informanformações e mecanismos de busca na web: panorama atual e tendências gerenciamento de conteúdo corporativo (ecm) na gestão de ti. Perspectivas em Ciência da Informação, 11(2), 161-173. doi: doi.org/10.1590/S1413-99362006000200002.

Supermercado Moderno. (2017). Ranking de supermercados. Recuperado de http://www.sm.com.br/ ranking-de-supermercados?tipo $=$ BuscaBrasil\&busca $=$ VERDEMAR\%20SUPERM.\%20E\%20PADARIA.
Verdemar. (2017). Documento recursos humanos: Manual treinamento de integração. treinamento e desenvolvimento. Documento não publicado.
Como citar este artigo (APA):

Tôrres, V. de A., Maia, L. C. G. \& Muylder, F. (2019). Criação de um chatbot para atendimento de suporte sistêmico de uma rede de supermercados a partir de uma base de conhecimento registrada no Mantis ${ }^{\circledR}$. AtoZ: novas práticas em informação e conhecimento, 8(2), 60 - 68. Recuperado de: http://dx.doi.org/10.5380/ atoz.v8i2.65170 